\title{
Shape Preserving Filament Enhancement Filtering
}

\author{
Michael H.F. Wilkinson and Michel A. Westenberg \\ Institute for Mathematics and Computing Science, \\ University of Groningen, P.O. Box 800, \\ 9700 AV Groningen, The Netherlands, \\ (michael, michel)@cs.rug.nl \\ http://www.cs.rug.nl/ michael/
}

\begin{abstract}
Morphological connected set filters for extraction of filamentous details from medical images are developed. The advantages of these filters are that they are shape preserving and do not amplify noise. Two approaches are compared: (i) multi-scale filtering (ii) single-step shape filtering using connected set (or attribute) thinnings. The latter method highlights all filamentous structure in a single filtering stage, regardless of the scale. The second approach is an order of magnitude faster than the first, filtering a $256^{3}$ volume in $41.65 \mathrm{~s}$ on a $400 \mathrm{MHz}$ Pentium II.
\end{abstract}

\section{Introduction}

Enhancement of curvi-linear, dendritic or other filamentous details has many applications in medical image analysis. Examples include computer analysed microscopy of filamentous microorganisms [9], confocal laser scanning microscopy of neurons, and various forms of angiography [4, 13]. Many methods have been proposed to enhance such details (for a review see e.g. 6]). Many of these methods have shortcomings, either in amplifying noise, or distorting certain important details, such as aneurisms or stenoses which may not be classified as filamentous features in some filters [6]. In particular, the use of linear scale spaces using Gaussian filters in multi-scale analysis can lead to distortion, merger, and movement of features in the image as more and more blurring is applied.

An entirely different issue is that of computational cost. Some of the more successful multi-scale approaches can be computationally very costly. For example, the multi-scale method of Sato et al. 8] requires 10 minutes of computations using eight $168 \mathrm{MHz}$ Sun Ultrasparc processors for an image of $256 \times 256 \times 102$ voxels, even when using just three scales. A similar method by Frangi et al. 4 is also done off-line due to the computational burden 13. Even if the approaches are not multi-scale, they often require repeated filtering with kernels sensitive to filaments running in different directions, which may mean combining 13 different directional filters [3].

The aim of this paper is to explore the possibilities offered by connected-set morphological filters in this context. Connected-set filters [5] have the distinguishing characteristic that they can enhance or remove an existing edge in an 
image, but never move it or introduce new edges. It is this edge, and therefore shape preserving property which offers the promise of vessel enhancement without changing vessel shapes, which is a prevalent problem in existing methods. Furthermore, because a great deal of progress has been made in the development of fast algorithms for these filters [1, 7, 12, filament extraction by connected filters is now a viable option.

We will first discuss the use of multi-scale morphological approaches, comparing classical morphological openings with their connected set counterparts: openings-by-reconstruction. One problem with these multi-scale methods is the repeated use of often costly filters: at least one for each scale. We therefore compare this approach with the use of shape based filters [10, which can extract all features of a given shape, regardless of their scale. These could find e.g. all filamentous structures in an angiogram in a single filtering stage, rather than by repeated operations as is done classically. It will be shown that operators with these characteristics can be found in the class of attribute operators [1, 7]. The discussion will focus on enhancing bright filamentous details only, because extension to dark details is trivial.

This paper mainly aims to show the speed gains of shape filters. A full-blown comparison with existing techniques will be performed later.

\section{Multi-scale Mathematical Morphology}

\section{$2.1 \quad$ Openings}

Extracting details at a particular scale using mathematical morphology can be done in a variety of ways, but the simplest is through the use of openings. An opening is a filter that removes bright or foreground details smaller than some particular scale from an image. A straightforward way to do this is by using grey scale erosions and dilations, also known as minimum and maximum filters, respectively. To perform an opening we first erode the image by assigning to each voxel the minimum grey value in a given neighbourhood (called structuring element). Next, the eroded image is dilated by assigning to each voxel the maximum grey value in the same neighbourhood. The size of the structuring element determines the scale of the details removed. In the following discussion, openings with spherical or cubic structuring elements of radius $r$ will be denoted by $\gamma_{r}^{B}$. In these openings the scale is determined by the local width of structures in the image.

Openings by structuring elements are not connected set filters, and therefore not shape preserving. An adaptation of these openings, called openings-byreconstruction [11] are connected set filters, and therefore shape preserving. An opening-by-reconstruction is performed by first eroding the image with a structuring element, and then reconstructing all details not completely removed by the erosion. In this way they remove image details completely, or leave them unaffected, but never change their shapes. Using structuring elements as above, the scale is again defined by width, but in this case it is the maximum width of a feature which determines the scale. 
A different class of openings, which use different scale parameters is the class of attribute openings [1]. The earliest of these was the area opening [2, which in 3 -D becomes a volume opening. In the binary case, a volume opening removes all connected foreground components with a volume smaller than some given threshold $\lambda$. If $T_{h}(f)$ denotes a binary image obtained by thresholding $f$ at grey level $h$, and $\Gamma_{\lambda}^{V}$ denotes the binary volume opening with scale parameter $\lambda$, the grey scale volume opening $\gamma_{\lambda}^{V}$ for image $f$ is given by:

$$
\left(\gamma_{\lambda}^{V}(f)\right)(x)=\sup \left\{h \mid x \in \Gamma_{r}^{V}\left(T_{h}(f)\right)\right\} .
$$

The interpretation of this equation is that the volume opening of an image assigns each point the highest threshold at which it still belongs to a connected foreground component $C$ with $V(C) \geq \lambda$, with $V(C)$ denoting the volume of $C$. This removes details in much the same way as the opening-by-reconstruction, but it uses the volume, rather than width as criterion.

In the following subsection we will introduce sets of openings called size distributions which can be used for filament extraction.

\subsection{Size Distributions}

A set of openings $\left\{\gamma_{r}\right\}$, in which $r$ is from some totally ordered set, with the property that

$$
\gamma_{r}\left(\gamma_{s}(f)\right)=\gamma_{\max (r, s)}(f)
$$

is called a size distribution or granulometry. All the openings described previously can be used to construct size distributions.

Suppose $r<s$. Obtaining an image $g$ containing all details from image $f$ within scale range $[r \ldots s)$ boils down to

$$
g=\gamma_{r}(f)-\gamma_{s}(f) \equiv \gamma_{r}(f)-\gamma_{s}\left(\gamma_{r}(f)\right)
$$

In words: remove everything smaller than $r$ from $f$, then remove all details smaller than $s$, and deduct the result from the opening at scale $r$. It does not matter whether we remove the details smaller than $s$ from the original image or from $\gamma_{r}(f)$.

\subsection{Multi-scale Filament Extraction}

If the scale parameter used is based on the width of the details, which is the case if we use $\left\{\gamma_{r}^{B}\right\}$ as size distribution, we can extract elongated details by removing all details with a volume smaller than some given threshold from $g$. If the scale $r$ defines the radius of the neighbourhood used in $\gamma_{r}^{B}$, objects with volumes larger than $\epsilon 8 r^{3}$, with $\epsilon \gg 1$ a measure of eccentricity of the object, are likely to be filamentous. Thin slabs of sufficient size will also be retained. Thus, the image $\gamma_{\epsilon 8 r^{3}}^{V}(g)$ will contain the filamentous details at scale $r$. It will retain straight, curved and forked filaments equally. 

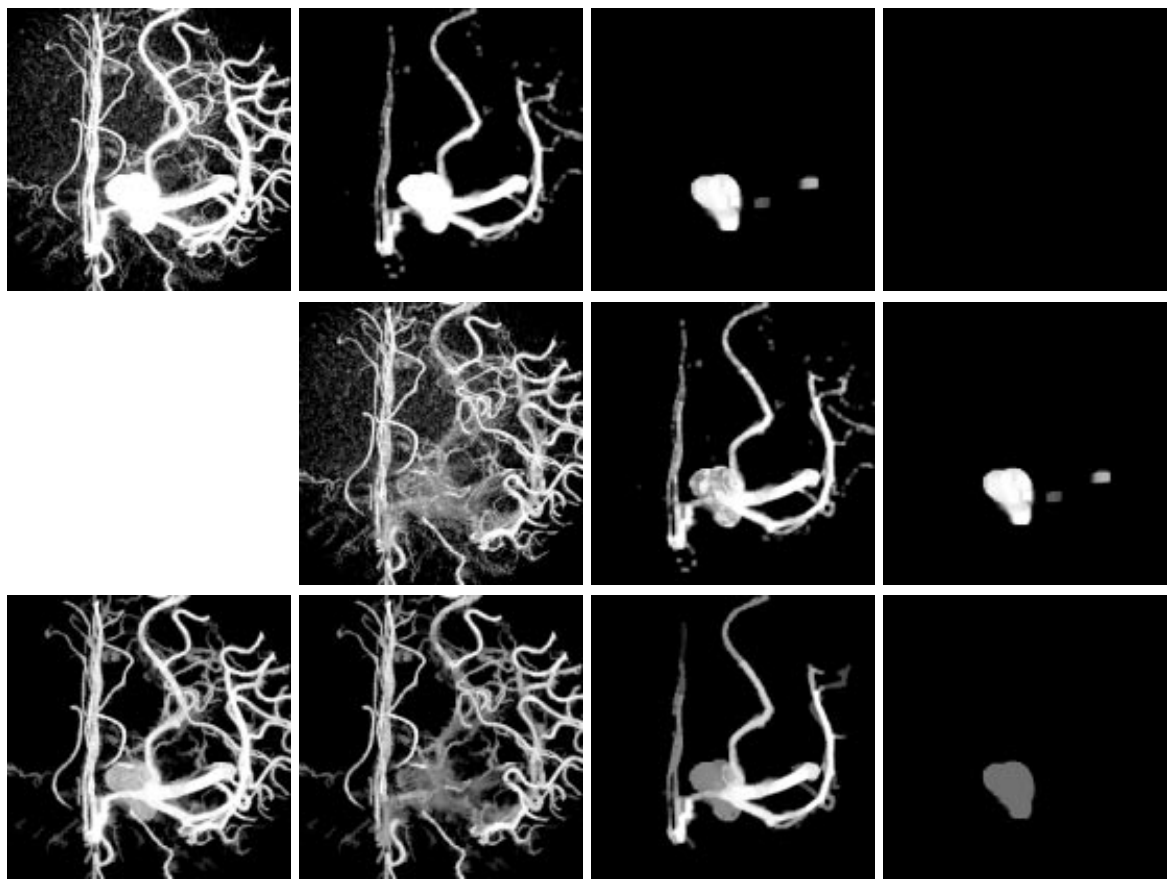

Fig. 1. Multiscale extractions of filamentous details using openings $\gamma_{r}^{B}$ at scales 1, 3 and 9 , using $\epsilon=4$. The leftmost column shows the original image (top) and final result (bottom). Each of the columns to the right show the process of extracting filamentous details at each of the scales. The top row contains the openings $\gamma_{r_{i}}^{B}$; the centre row the difference images with the opening at the previous scale (or original image at scale 1 ); the bottom row contains the volume opening $\gamma_{32 r_{i}}^{V}$ of the difference image.

We are now in a position to extract the filamentous details, starting at the thinnest, at $N$ different scales $r_{1}, r_{2}, \ldots, r_{N}$. The basic method is shown in Figure 1, which shows maximum intensity projections (MIP) of a $256 \times 256 \times 256$ rotational b-plane CT-angiogram (CTA) of the arteries of the right half of a human head. A contrast agent was used and an aneurism is present. In the first step, the original image $f_{0}$ (top left) is opened by $\gamma_{r_{1}}^{B}$ (second column, topmost). This image is subtracted from the original to extract the details at scales smaller than $r_{1}$ (second column, middle row), yielding an image we will denote as $f_{1}^{B}$. After this, we apply a volume opening $\gamma_{\epsilon 8 r_{1}}^{V}$ (second column, bottom row) to this image to obtain the filamentous detail at scale $r_{1}$. This image is denoted $f_{1}^{V}$.

At the next scales, we compute the opening $\gamma_{r_{i}}^{B}$, subtract this from $\gamma_{r_{i-1}}^{B}\left(f_{0}\right)$, to obtain detail image $f_{i}^{B}$, open that using $\gamma_{\epsilon 8 r_{i}}^{V}$ to obtain filament image $f_{i}^{V}$. This is shown for two higher scales in the third and fourth column. Once all $f_{i}^{V}$ are obtained, we sum them to obtain the output image (bottom left). 
Figure 1 shows that filamentous details are indeed extracted, but that the aneurism is processed after it has been separated from the vessels, and that its grey level is greatly reduced. In other words, it is not recognized as a clearly filamentous structure by this version of the algorithm. This is easily corrected by the use of connected set filters, which cannot separate the aneurism from the vessels. The multi-scale algorithm can be modified by replacing $\gamma_{r}^{B}$ by the equivalent openings-by-reconstruction. The result can be seen in Figure 2 Clearly, the opening-by-reconstruction retains the aneurism much better.

\subsection{Problems with the Multi-scale Approach}

One problem which arises is the quantization of the scale parameter. In the example shown in Figure 1 only three scales are used. In particular, there is a wide gap between scale $r_{2}=3$ and $r_{3}=9$. This means that image $f_{3}^{B}$ contains objects with widths ranging from $r=3$ to $r=8$. If such a fairly coarse quantization is used, elongated structures at the lower end of the range within one bin will be removed sooner than the thicker ones. This means that there will be a bias towards thicker structures in $f_{3}^{V}$. Ideally, we would want to filter at all scales, but this would be prohibitively costly. As it stands, performing a 3-scale filament extraction of a $256 \times 256 \times 256$ volume takes about $168 \mathrm{~s}$ on a $400 \mathrm{MHz}$ Pentium II processor (640 MB RAM) for the structural openings $\gamma_{r}^{B}$, and $192 \mathrm{~s}$ for openings-by-reconstruction. On a Compaq ES-40 (500 MHz DEC-Alpha) this takes $146 \mathrm{~s}$ and $131 \mathrm{~s}$ respectively. In general, the algorithm described here will have a computational cost proportional to the number of scales $N$. In the next section we will introduce a single step, connected set filtering approach, which effectively processes the image at all scales, without the computational cost.
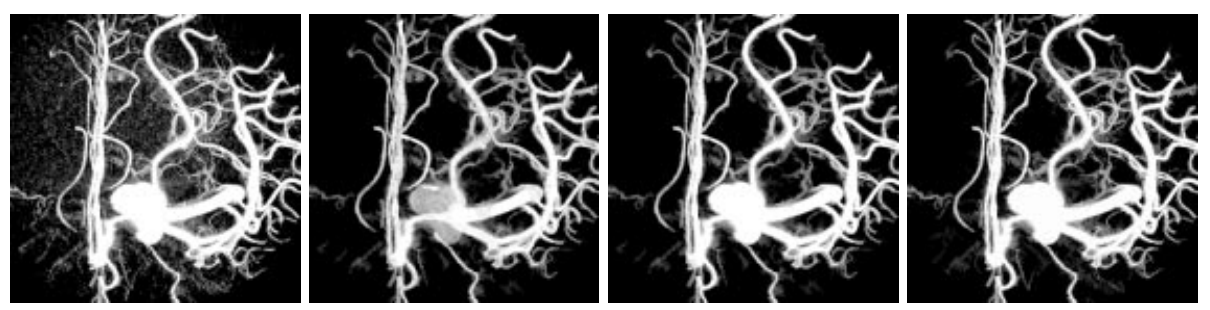

Fig. 2. A comparison of connected set filters and non-connected-set filters for filament extraction: from left to right: MIP of original image; result of multi-scale method using $\gamma_{r}^{B}$; result of multi-scale method using openings-by-reconstruction; result using attribute thinning $\phi_{2.0}^{S}$ as shape filter (see Section 3 ).

\section{Shape Filters}

A shape filter allows filtering based strictly on shape criteria, regardless of scale. As is shown in [10] attribute thinnings using the so-called subtractive rule provide such shape filters. This type of attribute thinning can be thought of as 
thresholding the original image at all possible grey levels, removing all connected foreground components of the wrong shape in each of the resulting binary images, and adding the resulting binary images up again. Selection of a particular shape category can be done by computing some shape number of each connected foreground component, and comparing it to some threshold value. The action of such an attribute thinning is shown in Figure 3. As can be seen, the thinning removes those features in the image which do not meet some shape criterion, whilst retaining all others. These filters can be computed efficiently for a variety of shape criteria using an adaptation of the Max-tree algorithm by Salembier et al. [7]. The only problem that remains is that of obtaining a shape criterion which can distinguish filamentous objects from all others.
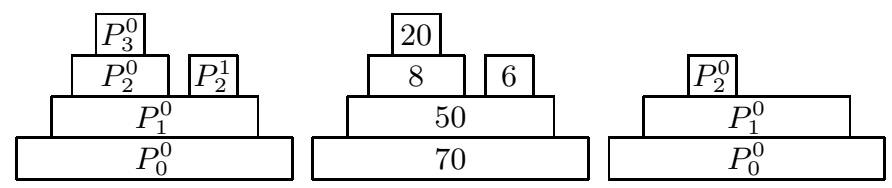

Fig. 3. The components $P_{j}^{i}$ of a grey level image $f$ (left), the corresponding attributes (middle) and the attribute thinning with a threshold $t=10$ (right).

The shape filter we propose is based on two different size criteria: the volume $V$ discussed above, and the moment of inertia $I$. For a given volume, the moment of inertia is minimal for a sphere, and increases rapidly as the object becomes more elongated. It can also be computed efficiently in the context of the Max-tree algorithm. For a connected set of pixels $C$ it is defined as

$$
I(C)=\frac{V(C)}{4}+\sum_{\mathbf{x} \in C}(\mathbf{x}-\overline{\mathbf{x}})^{2}
$$

in which $V(C)$ denotes the volume of $C$. The first term is required to account for the moment of inertia of individual voxels (which are assumed to be cubes). For a given 3-D shape, the moment of inertia scales with the size to the fifth power, whereas the the volume scales with the third power of the size. Therefore the ratio $S=\frac{I}{V^{5 / 3}}$ is a purely shape dependent number, i.e. it is scaling invariant, which has a minimum for a sphere (0.23) and increases rapidly with elongation. A thinning which extracts only components in which $S$ is larger than some threshold $t$ is denoted as $\phi_{t}^{S}$. An example of applying $\phi_{2.0}^{S}$ to the CTA used before is shown rightmost in Figure 2. The results are difficult to distinguish from the multi-scale connected set filter approach, but the thinning takes only $41.65 \mathrm{~s}$ to compute on a $400 \mathrm{MHz}$ Pentium II, and $33.15 \mathrm{~s}$ on a $500 \mathrm{MHz} \mathrm{DEC}$ Alpha. 


\section{Discussion}

Traditional structured openings such as $\gamma_{r}^{B}$ have a number of drawbacks for vessel extraction. Figure 4 shows a slice from the CTA cut through the aneurism, after filtering using the structured openings $\gamma_{r}^{B}$, and with the connected set shape filter $\phi_{2.0}^{S}$. The former method "hollows out" both the aneurism and thicker vessels: the central grey level is distinctly lower than that at the edge. This could pose severe problems for subsequent segmentation algorithms. By contrast, the connected set method does not suffer from this problem.
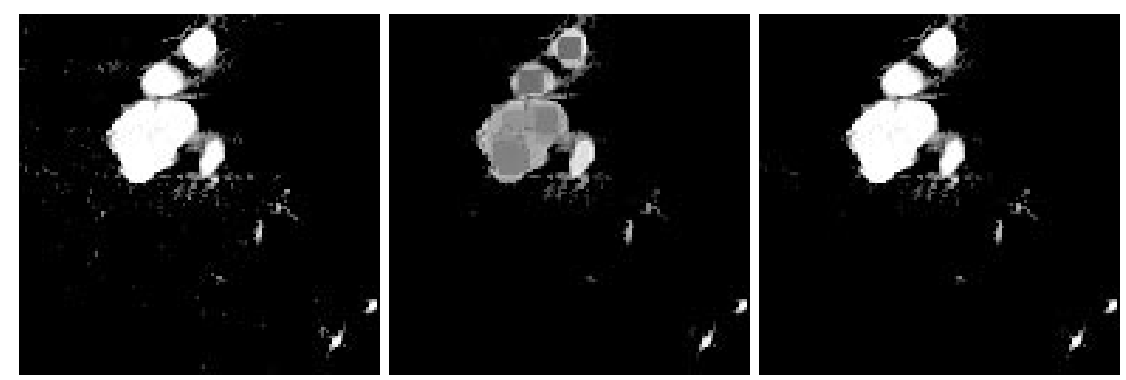

Fig. 4. Slice through CTA containing aneurism: (left) original; (middle) filtered using multi-scale approach with $\gamma_{r}^{B}$; (right) filtered using $\phi_{2.0}^{S}$. The middle image clearly shows a reduced grey level in the centre of the aneurism and some of the larger vessels. The connected filter approach does not suffer from this problem.

Figure 5 shows the results for $\phi_{2.0}^{S}$ applied to a phase contrast magnetic resonance angiogram (MRA) of $256 \times 256 \times 124$. The background is clearly suppressed, and the vessels are retained without distortion.
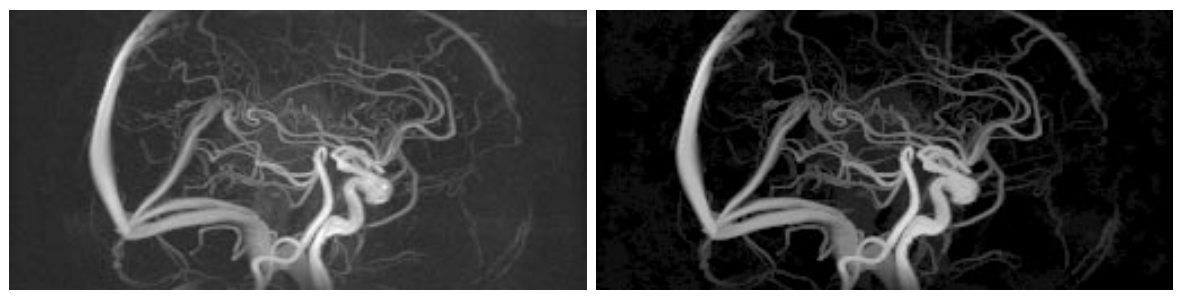

Fig. 5. MIP of MRA of a human brain: (left) original; (right) filtered using $\phi_{2.0}^{S}$. Filamentous detail is retained, whereas the background is suppressed.

It has been shown that connected-set filters can be used to extract filamentous details from images, either by a multi-scale approach, or by connected set shape filters. The latter approach is far faster, especially if many scales are needed. 
The great advantage of connected-set filters is their inability to move edges. This allows extraction of filamentous details without distortion, and without increasing noise. Of course, these filters are not perfect. There are many instances in which edges should be moved, e.g., to suppress boundary noise. This could be achieved by post-processing. It also remains to be seen whether connected-set shape filters improve segmentation. Finally, the attribute and threshold used in this shape filter may not be optimal, even though the performance in the images tested is encouraging. In the near future we intend to address these issues, and test shape filters against other methods systematically.

\section{References}

[1] E. J. Breen and R. Jones. Attribute openings, thinnings and granulometries. Computer Vision and Image Understanding, 64(3):377-389, 1996.

[2] F. Cheng and A. N. Venetsanopoulos. An adaptive morphological filter for image processing. IEEE Trans. Image Proc., 1:533-539, 1992.

[3] Y. P. Du and D. L. Parker. Vessel enhancement filtering in three-dimensional MR angiograms using long-range signal correlation. J. Magn. Reson. Imag., 7:447-450, 1997.

[4] A. F. Frangi, W. J. Niessen, K. L. Vincken, and M. A. Viergever. Multiscale vessel enhancement filtering. In W. M. Wells, A. Colchester, and S. Delp, editors, Medical Image Computing and Computer-Assisted Intervention - MICCAI'98, volume 1496 of Lecture Notes in Computer Science, pages 130-137. Springer, 1998.

[5] H. J. A. M. Heijmans. Connected morphological operators for binary images. Comput. Vis. Image Understand., 73:99-120, 1999.

[6] M. Orkisz, M. Hernández-Hoyos, P. Douek, and I. Magnin. Advances of blood vessel morphology analysis in 3D magnetic resonance images. Mach. Vis. Graph., 9:463-471, 2000.

[7] P. Salembier, A. Oliveras, and L. Garrido. Anti-extensive connected operators for image and sequence processing. IEEE Transactions on Image Processing, 7:555570, 1998.

[8] Y. Sato, S. Nakajima, N. Shiraga, H. Atsumi, S. Yoshida, T. Koller, G. Gerig, and R. Kinikis. 3D multi-scale line filter for segmentation and visualization of curvilinear structures in medical images. Medical Image Analysis, 2:143-168, 1998.

[9] A. Spohr, T. Agger, M. Carlsen, and J. Nielsen. Quantitative morphology of filamentous micro-organisms. In M. H. F. Wilkinson and F. Schut, editors, Digital Image Analysis of Microbes, pages 373-410. John Wiley and Sons, Ltd, Chichester, UK, 1998.

[10] E. R. Urbach and M. H. F. Wilkinson. Shape distributions and decomposition of grey scale images. IWI-report 2000-9-15, Institute for Mathematics and Computing Science, University of Groningen, 2001.

[11] L. Vincent. Morphological grayscale reconstruction in image analysis: application and efficient algorithm. IEEE Transactions on Image Processing, 2:176-201, 1993.

[12] M. H. F. Wilkinson and J. B. T. M. Roerdink. Fast morphological attribute operations using Tarjan's union-find algorithm. In Proceedings of the ISMM2000, pages 311-320, Palo Alto, CA, June 2000.

[13] O. Wink, W. J. Niessen, and M. A. Viergever. Fast delineation and visualization of vessels in 3-D angiographic images. IEEE Transactions on Medical Imaging, 19:337-346, 2000. 